\title{
Numerical verification for instrumented spherical indentation techniques in determining the plastic properties of materials
}

\author{
Taihua Zhang a) \\ State Key Laboratory of Nonlinear Mechanics (LNM), Institute of Mechanics, \\ Chinese Academy of Sciences, Beijing 100190, China \\ Peng Jiang \\ State Key Laboratory of Nonlinear Mechanics (LNM), Institute of Mechanics, \\ Chinese Academy of Sciences, Beijing 100190, China; and National Astronomical \\ Observatories, Chinese Academy of Sciences, Beijing 100012, China \\ Yihui Feng and Rong Yang \\ State Key Laboratory of Nonlinear Mechanics (LNM), Institute of Mechanics, \\ Chinese Academy of Sciences, Beijing 100190, China
}

(Received 16 June 2009; accepted 15 September 2009)

\begin{abstract}
Instrumented indentation tests have been widely adopted for elastic modulus determination. Recently, a number of indentation-based methods for plastic properties characterization have been proposed, and rigorous verification is absolutely necessary for their wide application. In view of the advantages of spherical indentation compared with conical indentation in determining plastic properties, this study mainly concerns verification of spherical indentation methods. Five convenient and simple models were selected for this purpose, and numerical experiments for a wide range of materials are carried out to identify their accuracy and sensitivity characteristics. The verification results show that four of these five methods can give relatively accurate and stable results within a certain material domain, which is defined as their validity range and has been summarized for each method.
\end{abstract}

\section{INTRODUCTION}

Over the past 10 years, a number of indentation-based methods have been proposed to measure plastic properties of materials. ${ }^{1-23}$ However, it is still contentious whether plastic properties can be accurately determined from an indentation test. For example, Cheng and Cheng ${ }^{2}$ have argued that the stress-strain curve may not be uniquely determined from loading and unloading curves by using the single sharp indenter method. In our opinion, rigorous verification is needed for these methods.

Recently, extensive assessment of the multiple-indenter method has been reported by Lan and Venkatesh. ${ }^{3}$ However, from the user's point of view, it is sometimes inconvenient to operate with distinct apex angle-indenters, which will add to the difficulty and cost of indentation tests. Recently, it has been shown that, with a spherical indenter, plastic properties can be determined from a single loading curve. ${ }^{18,19,23}$ By reviewing the spherical indentation methods that have been developed, ${ }^{7-23}$ the five models selected for this study were those of Field and Swain, ${ }^{14}$ Cao and Lu, ${ }^{18}$ Zhao et al., ${ }^{19}$ Lee, ${ }^{11}$ and Jiang et al. ${ }^{23}$ Although there have certainly been many more studies performed on this

\footnotetext{
a) Address all correspondence to this author.

e-mail: zhangth@lnm.imech.ac.cn

DOI: 10.1557/JMR.2009.0428
}

topic, these five were selected for three reasons. First, they can be simply reproduced step by step according to only the procedures provided by the original literature; second, they offer a means of identifying the plastic properties solely by analyzing the load-depth data (that is, they do not rely on the physical measurement of residual impression); third, they are easy to implement experimentally (no difficulty is caused by repetitive loading and unloading, and only a small number of empirical parameters are used).

Although several experimental verifications have been done, they have often given totally different results for the same model. For example, verification by Guelorget et al. ${ }^{24}$ with an 8.1- $\mu \mathrm{m}$-radius indenter has shown good performance for the method of $\mathrm{Cao}$ and $\mathrm{Lu} .{ }^{18}$ However, for the same method, a contrary conclusion was reached by Herbert et al. ${ }^{25}$ using an indenter with $385-\mathrm{nm}$ radius. It is difficult to assess which verification result is more reasonable, because different experimental conditions will obviously affect the accuracy of test results.

To clarify these issues, in this study, we performed numerical verification for a wide range of materials, which were free from effects of experimental conditions. In addition, the effectiveness of our computation model is proven by our previous test data. ${ }^{23}$ With these numerical results, the accuracy of each model was studied. Furthermore, 
considering that the sensitivity characteristic has been recognized as another important factor for the evaluation of indentation-based methods, stability analysis of these five models was also performed. Finally, within specified accuracy and stability criteria, the validity range of each method was quantified.

\section{BACKGROUND OF THE MODELS}

Without losing generality, the elastic-Hollomon's powerlaw hardening assumption was adopted in these models; that is, the uniaxial stress-strain $(\sigma-\varepsilon)$ curve of material was expressed in the form

$$
\left\{\begin{array}{ll}
\sigma=E \varepsilon, & \text { for } \quad \varepsilon \leq \varepsilon_{y} \\
\sigma=E \varepsilon_{y}^{1-n} \varepsilon^{n}, & \text { for } \quad \varepsilon \geq \varepsilon_{y}
\end{array},\right.
$$

where $E$ is the elastic modulus, $\sigma_{y}$ is the yield stress, $n$ is the strain hardening exponent, and $\varepsilon_{y}$ is equal to the ratio of yield stress to elastic modulus. Such a material model has been proven to describe well the stress-strain curves of most metals. ${ }^{26}$

\section{A. Method I: Model of Field and Swain}

The critical component of the model developed by Field and Swain ${ }^{14}$ is based on the assumption that, if a material obeys power law hardening, it is possible to use the work-hardening index, $n$, to account for the "piling-up" or "sinking-in" of the contact perimeter as follows.

$$
c^{2}=\frac{5}{2}\left(\frac{2-n}{4+n}\right)
$$

where $c=a_{c} / a$. Furthermore, Hill et al. ${ }^{27}$ have shown, in particular for spherical indentation of power-law solids, that self-similarity is at hand, and solutions for moving contact can be generated from a stationary solution by an appropriate scaling procedure. In this spirit, they provided a rigorous solution as follows

$$
\frac{F}{\pi a_{c}^{2}}=k\left(\frac{a_{c}}{2 R}\right)^{n}
$$

where $F$ is indentation force, $a_{c}$ is the contact radius, and $\alpha$ and $\beta$ are universal constants. ${ }^{9}$ Field and Swain ${ }^{14}$ rearranged their Eqs. (2) and (3) by taking logarithms

$$
\log F=\log \left(\frac{\pi \kappa c^{2+n}}{2^{n} R^{n}}\right)+(2+n) \log a
$$

Linear regression of $\log F$ on $\log a$ gave $2+n$ as the gradient. Hence, by using Tabor's relation $\left(\sigma_{r}=H / 2.8\right.$ and $\left.\varepsilon_{r}=0.2 a_{c} / R\right){ }^{7}$ a representative stress-strain curve can be derived without having to rely on physical measurements of the residual impression. Although the method of Field and Swain ${ }^{14}$ does not specifically identify the yield strength, knowing the elastic modulus allows us to estimate the yield strength from the intersection of the elastic and power law stress-strain relations.

\section{B. Method II: Model of Cao and Lu}

For spherical indentation, dimensional analysis leads to

$$
\frac{F}{h^{2} \sigma_{r}}=\prod\left(\frac{h}{R}, \frac{\sigma_{r}}{E}, n\right) .
$$

By extending the representative strain as defined in the work of Dao and Cholloop ${ }^{28}$ for sharp indentation to spherical indentation, a representative method was developed by $\mathrm{CaO}$ and $\mathrm{Lu} .{ }^{18}$ In this model, the representative strain is introduced to reduce the number of apparent unknown variables to one. For a given indentation depth $h / R$, there exists a representative strain $\varepsilon_{r}$ and corresponding representative stress $\sigma_{r}$ (Fig. 1), from which $\sigma_{r}$ can be expressed as

$$
\begin{aligned}
\frac{F}{\sigma_{r} h^{2}}= & M_{1} \ln ^{3}\left(\frac{E_{r}}{\sigma_{r}}\right)+M_{2} \ln ^{2}\left(\frac{E_{r}}{\sigma_{r}}\right) \\
& +M_{3} \ln \left(\frac{E_{r}}{\sigma_{r}}\right)+M_{4},
\end{aligned}
$$

where the coefficients $M_{1}, M_{2}, M_{3}$, and $M_{4}$ depend on relative indentation depth $h / R$ (Table I), $E_{r}=E /\left(1-v^{2}\right)$ is the reduced modulus, and $\varepsilon_{r c}$ satisfies the relation

$$
\begin{aligned}
\varepsilon_{r c}= & 0.00939+0.435 \frac{h}{R} \\
& -1.106\left(\frac{h}{R}\right)^{2}\left(0.01 \leq \frac{h}{R} \leq 0.1\right) .
\end{aligned}
$$

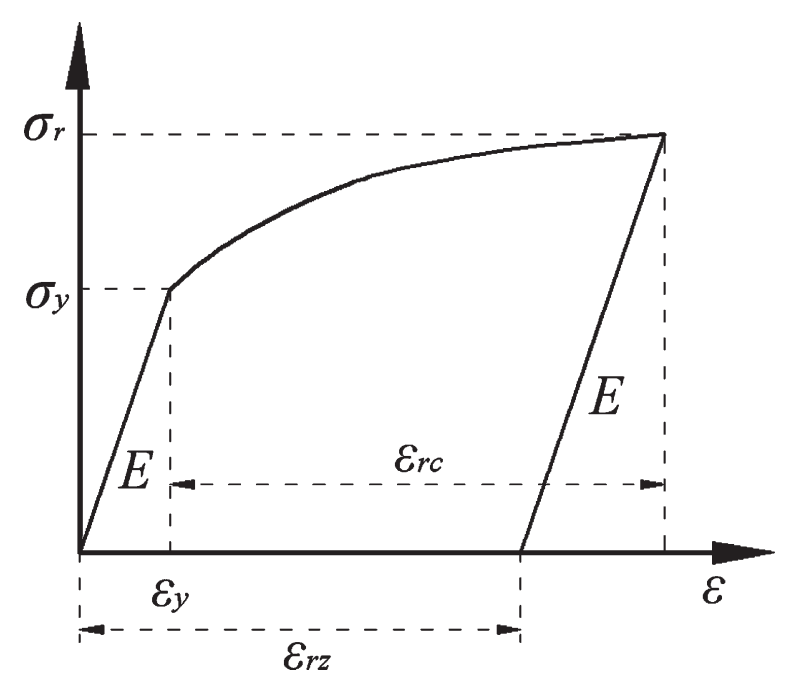

FIG. 1. A schematic of representative strain, where $\varepsilon_{\mathrm{rc}}$ and $\varepsilon_{\mathrm{rz}}$ are, respectively, the representative strains defined by $\mathrm{CaO}$ and $\mathrm{Lu}^{18}$ and Zhao et al. ${ }^{19}$ 
TABLE I. Coefficients of Eq. (7) corresponding to various $h / R^{[41]}$.

\begin{tabular}{lrrrr}
\hline \hline$h / R$ & $\mathrm{M}_{1}$ & $\mathrm{M}_{2}$ & \multicolumn{1}{c}{$\mathrm{M}_{3}$} & \multicolumn{1}{c}{$\mathrm{M}_{4}$} \\
\hline 0.01 & -21.835 & 331.125 & -1097.352 & 1155.349 \\
0.06 & -3.236 & 36.951 & -48.125 & 7.134 \\
\hline \hline
\end{tabular}

Here the representative strain is defined as the nonlinear part of the effective strain accumulated beyond the yield strain, that is, $\varepsilon_{r c}=\varepsilon-\sigma_{y} / E$ (Fig. 1).

If the elastic modulus of the indented material is known, from the spherical indentation load at two specific relative indentation depths $(0.01 R$ and $0.06 R$ are suggested by $\mathrm{Cao}$ and $\mathrm{Lu})$, two data points $\left(\varepsilon_{r}, \sigma_{r}\right)$ can be determined from Eqs. (6) and (7). Thus, the plastic properties, i.e., yield strength and the stain hardening exponent, can be uniquely determined using Eq. (1) with known elastic modulus.

\section{Method III: Model of Zhao et al.}

The method proposed by Zhao et al. ${ }^{19}$ chooses another two indentation depths $(h / R=0.13$ and $h / R=0.30)$. By adjusting the representative strain and stress, the best fit of Eq. (5) is achieved such that the apparent dependence of $n$ is minimum. For $h / R=0.13, \varepsilon_{r z l}=0.0374$; for $h / R=$ $0.30, \varepsilon_{r z 2}=0.0674$. Here the representative strain is defined as $\varepsilon_{r z}=\varepsilon-\sigma_{r} / E$ (Fig. 1), which is somewhat different from that of method II. With these two indentation depths, the fitting functions take the following form.

$$
\left\{\begin{array}{lll}
\frac{F}{\sigma_{r 1} h^{2}}=f_{1}\left(m_{1}\right) \times k_{1}(n), & \frac{h}{R}=0.13 \\
\frac{F}{\sigma_{r 2} h^{2}}=f_{2}\left(m_{2}\right) \times k_{2}(n), & \frac{h}{R}=0.30
\end{array} .\right.
$$

For $h / R=0.13$

$$
\begin{aligned}
f_{1}\left(m_{1}\right)= & 32.77-52.59 \ln \left(m_{1}\right)+33.46 \ln ^{2}\left(m_{1}\right) \\
& -4.8000 \ln ^{3}\left(m_{1}\right)+0.2147 \ln ^{4}(m),
\end{aligned}
$$

and

$$
\begin{aligned}
k_{1}(n)= & 1.001+0.2610 n-0.5217 n^{2} \\
& +0.1547 n^{3} .
\end{aligned}
$$

For $h / R=0.30$

$$
\begin{aligned}
f_{2}\left(m_{2}\right)= & 3.817-12.73 \ln \left(m_{2}\right)+11.99 \ln ^{2}\left(m_{2}\right) \\
& -2.032 \ln ^{3}\left(m_{2}\right)+0.1049 \ln ^{4}\left(m_{2}\right),
\end{aligned}
$$

and

$k_{2}(n)=1.002+0.7637 n-0.1920 n^{2}+1.255 n^{3}$,

where $m_{1}=E_{r} / \sigma_{r 1}$ and $m_{2}=E_{r} / \sigma_{r 2}$, and $\sigma_{r 1}$ and $\sigma_{r 2}$ are the representative stresses corresponding to $\varepsilon_{r 1}=0.0374$ and $\varepsilon_{r 2}=0.0674$, respectively.
For a given spherical indentation test, $F / h^{2}$ corresponding to two certain indentation depths $(h / R=0.13$ and $h / R=0.30)$ can be determined by experiment. Thus, by varying the unknown variables $\left(\varepsilon_{y}, n\right)$ in a large range, the errors $\left[e_{1}=F_{1} / \sigma_{r 1} h_{1}^{2}-f_{1}\left(m_{1}\right) k_{1}(n)\right.$, $\left.e_{2}=F_{2} / \sigma_{r 2} h_{2}^{2}-f_{2}(m) k_{2}(n)\right]$, can be calculated for every set of material properties with known elastic modulus, where $f_{1}, k_{1}, f_{2}$, and $k_{2}$ are given by Eqs. (9)-(12). Finding the least total error $e=\left|e_{1}\right|+\left|e_{2}\right|$ will lead to the solution of Eq. (8) (or an output close to the solution). However, the resolution of such output results depends on the intervals of plastic properties adopted in the searching range. In the present verification, $\varepsilon_{y}$ increased from 0.0001 to 0.06 with a $1 \%$ rate of increase, and $n$ increased from 0 to 0.5 in increments of 0.01 , which ensured that the output result was sufficiently close to the analytical solution of Eq. (8).

\section{Method IV: Model of Lee}

Unlike the previous work, Lee ${ }^{11}$ selected a reference point that was $<10 \%$ of the indenter diameter from the surface and $0.8 a$ from the indentation center. The study of Lee ${ }^{11}$ shows that, at this selected point, equivalent strain was free from frictional effects and could be expressed as

$$
\begin{aligned}
\varepsilon & =f_{0}^{\varepsilon}(n) f_{2}^{\varepsilon}\left(\varepsilon_{y}\right)\left(\frac{a_{c}}{R}\right)^{f_{1}^{\varepsilon}(n) f_{3}^{\varepsilon}\left(\varepsilon_{y}\right)} \\
f_{0}^{\varepsilon}(n) & =1.82 n-5.82 n^{2}+6.92 n^{3} \\
f_{1}^{\varepsilon}(n) & =1.45 n-0.64 n^{2}-0.233 n^{3} \\
f_{2}^{\varepsilon}\left(\varepsilon_{y}\right) & =1.05 \varepsilon_{y}-19.2 \varepsilon_{y}^{2}-3850 n^{3} \\
f_{3}^{\varepsilon}\left(\varepsilon_{y}\right) & =0.895 \varepsilon_{y}+66.7 \varepsilon_{y}^{2}-7090 \varepsilon_{y}^{3} .
\end{aligned}
$$

Furthermore, the corresponding equivalent stress can be written as

$$
\sigma=\frac{F}{\pi a_{c}^{2} \psi}
$$

$\Psi$ can be expressed as

$$
\begin{aligned}
\psi & =f_{0}{ }^{\psi}(n) f_{2}{ }^{\psi}\left(\varepsilon_{y}\right)+f_{1}{ }^{\psi}(n) f_{3}{ }^{\psi}\left(\varepsilon_{y}\right)\left(\frac{a_{c}}{R}\right) \\
f_{0}{ }^{\psi}(n) & =3.06 n-4.4 n^{2}+4.19 n^{3} \\
f_{1}{ }^{\psi}(n) & =0.227 n-0.317 n^{2}+1.25 n^{3} \\
f_{2}{ }^{\psi}\left(\varepsilon_{y}\right) & =1.06 \varepsilon_{y}-30.3 \varepsilon_{y}^{2}-307 n^{3} \\
f_{3}{ }^{\psi}\left(\varepsilon_{y}\right) & =3.34 \varepsilon_{y}-1290 \varepsilon_{y}^{2}+61000 \varepsilon_{y}^{3},
\end{aligned}
$$

and $a_{c}$ can be determined by

$$
a_{c}=\sqrt{2 c^{2} h R-\left(c^{2} h\right)^{2}},
$$


where

$$
\begin{aligned}
c^{2} & =f_{0}^{c}(n) f_{2}^{c}\left(\varepsilon_{y}\right)+f_{1}^{c}(n) f_{3}^{c}\left(\varepsilon_{y}\right) \ln \left(\frac{50 h_{t}}{R}\right) \\
f_{0}^{\psi}(n) & =1.09 n-1.21 n^{2}+1.13 n^{3} \\
f_{1}^{\psi}(n) & =0.104 n-0.323 n^{2}+0.405 n^{3} \\
f_{2}{ }^{\psi}\left(\varepsilon_{y}\right) & =1.19 \varepsilon_{y}-117 \varepsilon_{y}^{2}+11500 n^{3} \\
f_{3}{ }^{\psi}\left(\varepsilon_{y}\right) & =0.508 \varepsilon_{y}+345 \varepsilon_{y}^{2}-49500 \varepsilon_{y}^{3} .
\end{aligned}
$$

By initially guessing values of $\varepsilon_{y}$ and $n, c^{2}, \varepsilon_{p}$, and $\sigma$ can be derived by the above equations for each loaddepth data point on the load-depth curve. Consequently, the values of $\varepsilon_{y}$ and $n$ can be calculated from the stressstrain relation [Eq. (1)]. Then the values of $\varepsilon_{y}$ and $n$ are updated, and repeatedly calculated until the updated $\varepsilon_{y}$ and $n$ are convergent within the tolerance.

\section{E. Method V: Model of Jiang et al.}

By considering the effect of piling-up on the volume displaced by hydrostatic core, a modified expanding cavity model (ECM) was developed in our previous study $^{23}$ to describe the relationship between indentation work and plastic properties as follows

$$
\begin{aligned}
W_{t}= & \frac{2 \pi E \varepsilon_{y}^{2} c^{3}}{3 n(n+1)}\left[\left(\frac{c}{a}\right)^{3 n}-1\right] \\
& +\frac{(n-1) \pi E \varepsilon_{y}^{2} a^{3}}{3(n+1)}\left[\left(\frac{c}{a}\right)^{3}-1\right]+\frac{\pi E \varepsilon_{y}^{2} c^{3}}{3},
\end{aligned}
$$

where $c=\kappa \sqrt[3]{\frac{h^{2}(3 R-h)}{3 \varepsilon_{y}}}$, and $\kappa$ is a correction factor considering the piling-up effect, which is given as

$$
\begin{aligned}
\kappa= & f\left(\varepsilon_{y}, n\right)=\left(-0.0077 n^{2}+0.0534 n-0.0304\right) \log ^{2}\left(\varepsilon_{y}\right) \\
& +\left(0.3717 n^{2}-0.1331 n-0.0774\right) \log \left(\varepsilon_{y}\right) \\
& +0.495 n^{2}-0.3016 n+1.0627 .
\end{aligned}
$$

On the other hand, the similarity solution was further studied numerically in detail. Then, within the fixed range $0.6 \leqslant a / R \leqslant 0.7$, the relation between the Meyer index and plastic properties was established as follows

$$
\begin{aligned}
m= & \left(-792.59 n^{2}+1675.9 n-962.01\right) \varepsilon_{y}^{2} \\
& +\left(68.187 n^{2}-112.78 n+57.84\right) \varepsilon_{y} \\
& -1.4569 n^{2}+2.8637 n+1.7178,
\end{aligned}
$$

where the Meyer index is the gradient of the linear regression of $\log F$ on $\log a$.

For a given spherical indentation test with specific range $h / R$ (from 0 to $0.3 R$ ), $W_{t}$ and $m$ can be obtained from the load-depth curve. $E$ is taken as a previously known parameter. Using the Newton iteration method, Eqs. (18) and (20) can be solved to obtain the plastic parameters $\sigma_{y}\left(\sigma_{y}=E \varepsilon_{y}\right)$ and $n$.

\section{NUMERICAL VERIFICATION}

Finite element computations were carried out using ABAQUS to verify these methods. The spherical indenter was assumed to be rigid. The Coulomb friction law was applied to the contact surfaces, the friction coefficient was taken to be $0.15,{ }^{19}$ and Poisson's ratio was fixed at 0.3 . Both of the above parameters are minor factors of indentation. A typical mesh for the axisymmetric indentation model comprised $>10,000$-node elements with reduced integration.

To verify the computation model, the measured loaddepth curves ${ }^{23}$ of steel $45 \mathrm{C}, \mathrm{Al} 6061$, and steel IF were compared with simulated load-depth curves obtained by introducing in finite element analysis (FEA) the true stress-true strain curve. The indentation experiments were performed using an MTS Nano Indenter XP; the radius of the spherical indenter was $10.8 \mu \mathrm{m} .{ }^{23}$ As shown in Fig. 2, the experimental and FE-simulated load-depth curves were in a fairly good agreement, thus showing the capability of the FE model to simulate the indentation process with satisfactory accuracy.

Materials with plastic properties within the ranges $\varepsilon_{y 0}$ varying from 0.0003 to 0.03 , and $n_{0}$ between 0 and 0.5 , were simulated so that most engineering materials were covered. In direct application of these methods, the estimated plastic properties $\left(\varepsilon_{y}, n\right)$ can be derived by processing the simulation results of each material combination $\left(\varepsilon_{y 0}, n_{0}\right)$. By comparing the values of $\left(\varepsilon_{y}, n\right)$ and $\left(\varepsilon_{y 0}, n_{0}\right)$, we found that method (I) failed to provide satisfactory estimations. That result was not surprising because it has been proven that Tabor's relation, the foundation of the Field and Swain model, has no direct correlation with the material properties, ${ }^{12}$ and the Meyer index cannot be simply described as $n+2$. $^{23}$

Herbert et al. ${ }^{25}$ has suggested that the constraint factor in Tabor's relation should not be 2.8 but 3.7 , which leads to a good predicted result for $\mathrm{Al}$ 6061. However, by

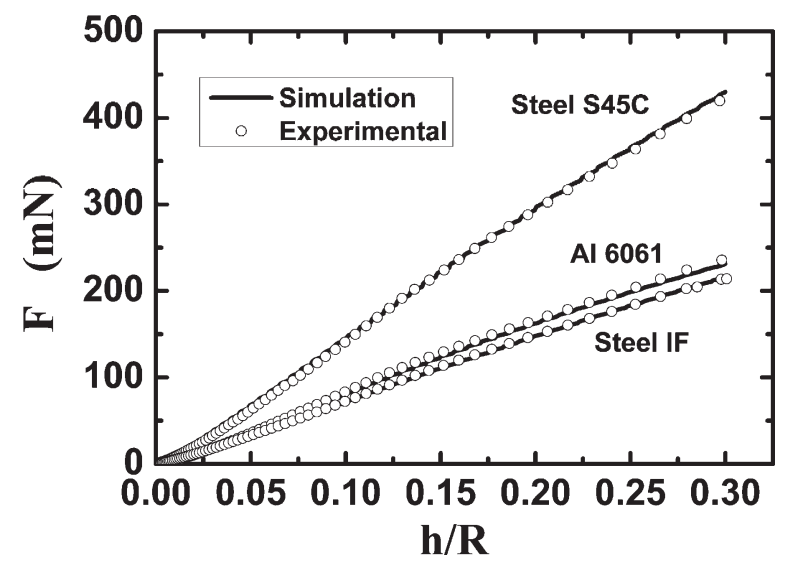

FIG. 2. Comparison of numerical and experimental indentation curves for three typical metals, showing the capability of the FE model for simulating the indentation process with satisfactory accuracy. 
adopting this suggestion in our numerical verification, we still could not find a correlation between predicted results and exact plastic properties.

For other models, the relative errors of the yield strength $\left(\sigma_{y}-\sigma_{y 0}\right) / \sigma_{y 0}$ and absolute errors of the work hardening

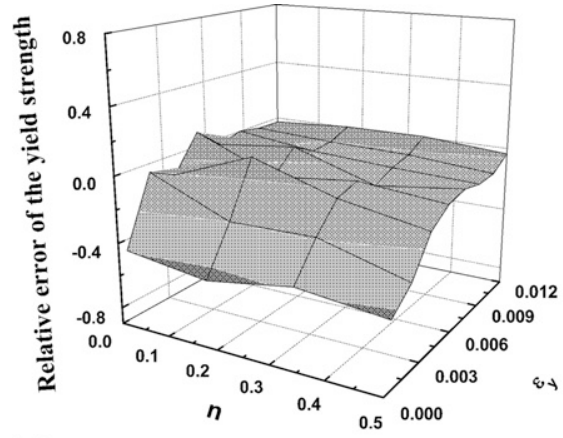

(a)

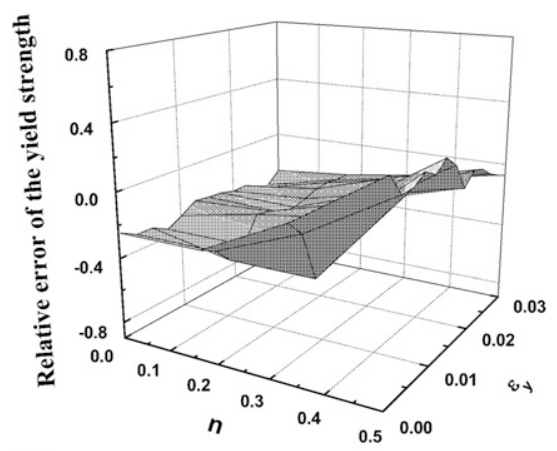

(c)

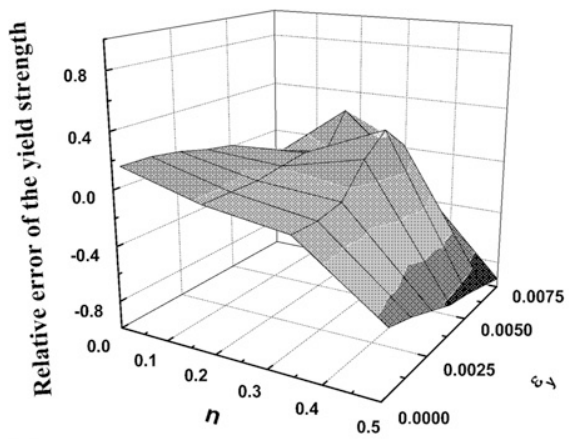

(e)

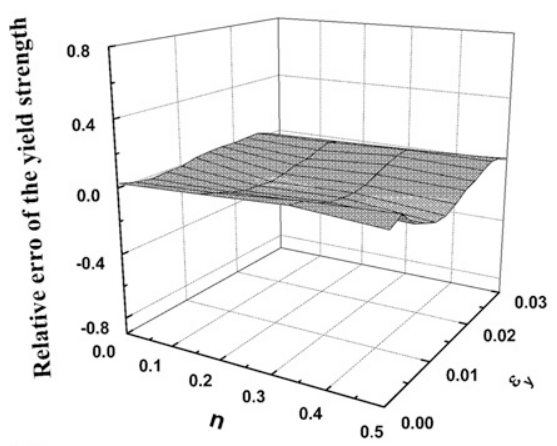

(g) exponent $\left(n-n_{0}\right)$ are presented in Fig. 3. $\sigma_{y 0}$ and $n_{0}$ represent the input parameters of the simulation, and $\sigma_{y}$ and $n$ represent the predicted results of these methods.

As shown in Figs. 3(a) and 3(b), method (II) can provide a unique solution only for materials within the

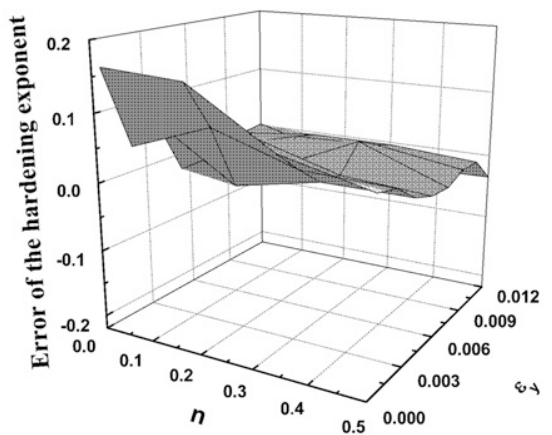

(b)

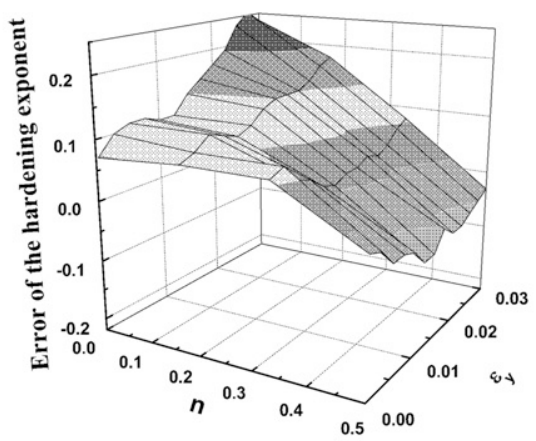

(d)

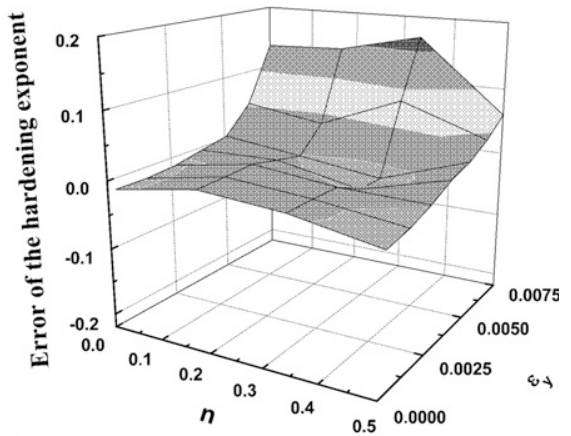

(f)

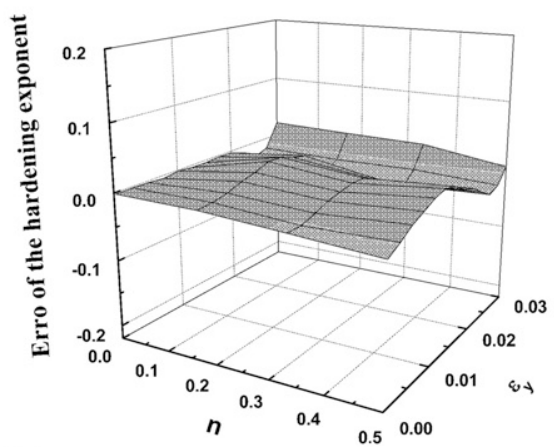

(h)

FIG. 3. The relative errors of the yield strength and absolute errors of the hardening exponent of (a, b) method (II); (c, d) method (III); (e, f) method (IV); and (g, h) method (V). 
ranges $0.0005 \leqslant \varepsilon_{y} \leqslant 0.0120$ and $0 \leqslant n \leqslant 0.5$. The maximum error of the yield strength predicted by method II was $\sim 60 \%$, and in most cases, the error was $<20 \%$. The maximum error of the hardening exponent was $\sim 0.2$, and in most cases, the error was $<0.1$. It was also found that with decrease of $\varepsilon_{y}$, the predicted error tended to increase. This may be because we ignored friction effects that have been proven to have an obvious effect on the load-depth curves for piling-up materials, which always correspond to small $\varepsilon_{y}$.

For method (IV), a unique solution could be derived only for the even smaller ranges $0.0005 \leqslant \varepsilon_{y} \leqslant 0.0075$ and $0 \leqslant n \leqslant 0.5$. The maximum error of the yield strength predicted by method IV was $\sim 90 \%$, and in most cases, the error was $<30 \%$ [Fig. 3(e)]. The maximum error of the hardening exponent was $\sim 0.2$ and in most cases was $<0.05$ [Fig. 3(f)].

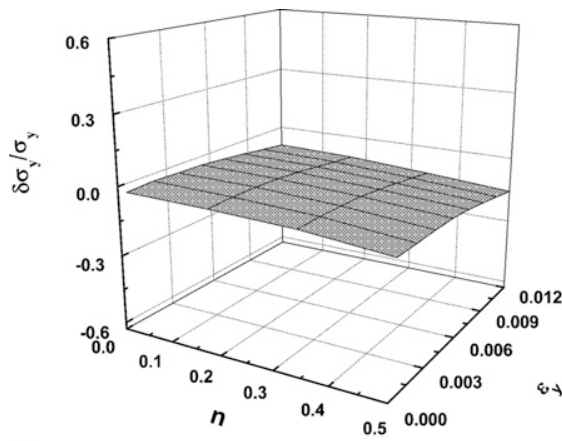

(a)

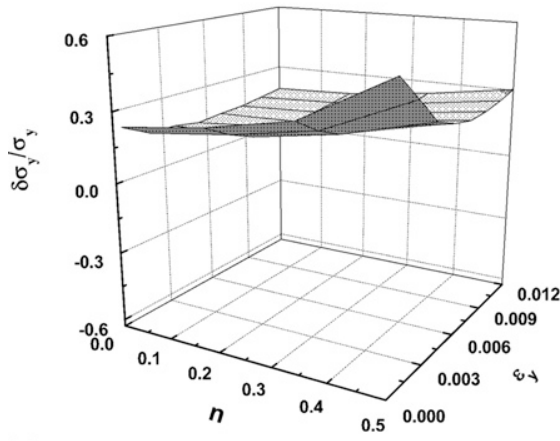

(c)

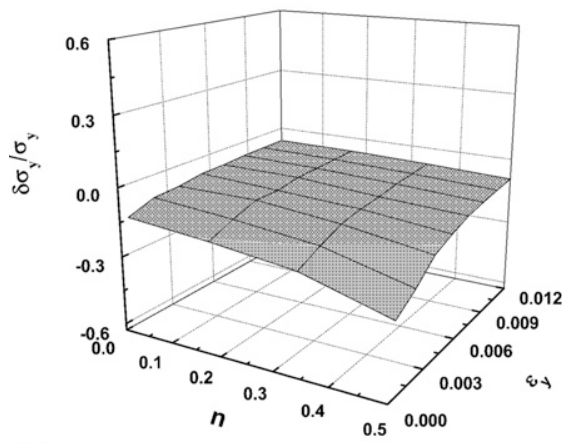

(e)

(d)

(b)

Although both methods (III) [Figs. 3(c) and 3(d)] and (V) [Figs. 3(g) and 3(h)] can give a unique solution for all materials that were simulated in this work, the maximum errors of the yield strength predicted by methods (III) and (V) were, respectively, $\sim 40$ and $10 \%$ and in most cases were $<20$ and $<5 \%$, respectively. The maximum errors of the hardening exponent were $\sim 0.25$ and 0.05 , respectively, and mostly $<0.15$ and $<0.02$, respectively.

For the indentation-based methods to be widely adopted in extracting plastic properties, they must be proven to be sufficiently robust that small uncertainties or variations in the measured indentation response parameters, that could arise because of factors associated with the test instrumentation, test environment, or test material, do not lead to unreasonably high variability in the estimation of material properties. Consequently, the stability of these proposed methods should be studied in greater detail.
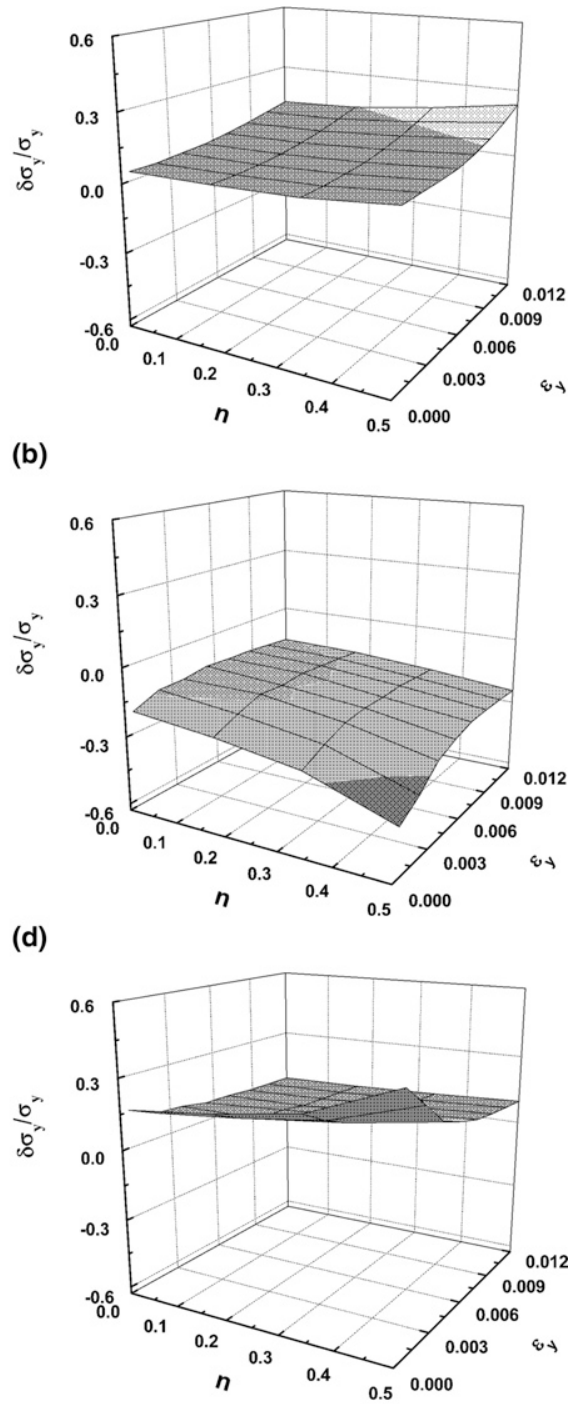

(f)

FIG. 4. Sensitivity distribution observed in determination of the yield stress of method (III) with uncertainties of (a, b) $+4 \%$ and $-4 \%$ in $E$; (c, d) $+3 \%$ and $-3 \%$ in $F_{0.13}$; and (e, f) $+3 \%$ and $-3 \%$ in $F_{0.30}$. 
To characterize the sensitivity of these proposed indentation-based methods in determining the plastic properties of materials, independent relative perturbations were introduced into indentation responses, e.g., $W_{t}, m, F$, and $E$. Following an early suggestion of Cao and $\mathrm{Lu}^{18}$ and Oliver and Pharr, ${ }^{30}$ the relative perturbation of $E$ in this sensitivity analysis was set at $4 \%$, and the relative perturbations of $W_{t}, F$, and $m$ were determined to be 3,3 , and $2 \%$, respectively, according to the dispersion degree of our previous physical test data. ${ }^{23}$ After introducing these perturbation levels in response parameters, the inverse approaches outlined in Sec. II were used to derive the plastic properties $\sigma_{y} \phi$ and $n \phi$. The deviation of yield stress $\left[\left(\sigma_{y}{ }^{\prime}-\sigma_{y 0}\right) / \sigma_{y 0}\right]$ determined by each model was presented as a threedimensional map (Fig. 4).

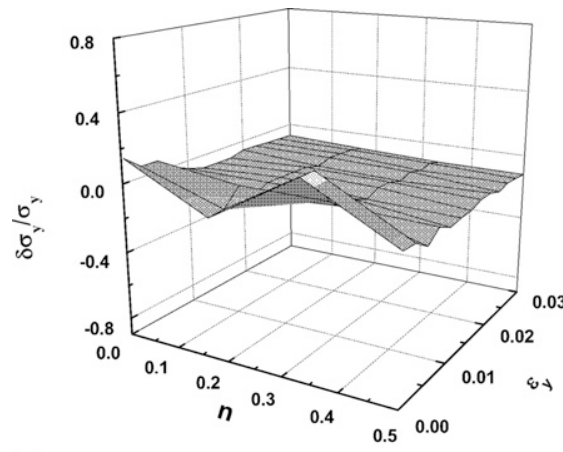

(a)

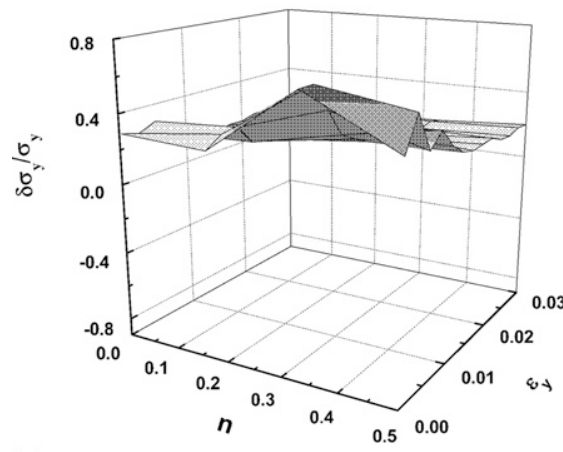

(c)

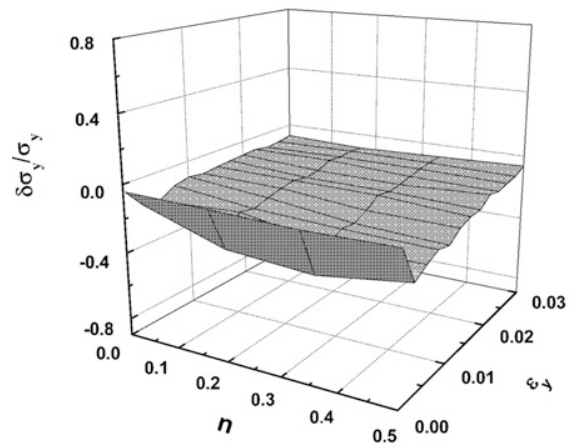

(e)

FIG. 5. Sensitivity distribution observed in determination of the yield stress of method (II) with uncertainties of (a, b) $+4 \%$ and $-4 \%$ in $E$; (c, d) $+3 \%$ and $-3 \%$ in $F_{0.01}$; (e, f) and $+3 \%$ and $-3 \%$ in $F_{0.06}$.
As shown in Fig. 5, the yield strength derived by method (II) is most sensitive to the uncertainties of $F_{0.01}$. The maximum deviation of yield strength caused by the uncertainties of $F_{0.01}$ is $\sim 55 \%$, which corresponds to $\varepsilon_{y}=0.0003$ and $n=0.5$, and the deviation of yield strength decreases with increasing $\varepsilon_{y}$ or decreasing $n$; in most cases, these deviations are $<30 \%$.

Similar, the highest sensitivity of method (III) was found for uncertainties of $F_{0.13}$ (Fig. 4), which corresponds to $h=0.13 R$. The maximum deviation is $\sim 60 \%$ and is mostly $<30 \%$. The distribution rule of the yield strength derivations is also similar to that of method (II).

For method (IV), it is difficult to verify the sensitivity character for each indentation depth, because too many load-depth test data points were adopted in this method. The perturbation of indentation loads that we introduced

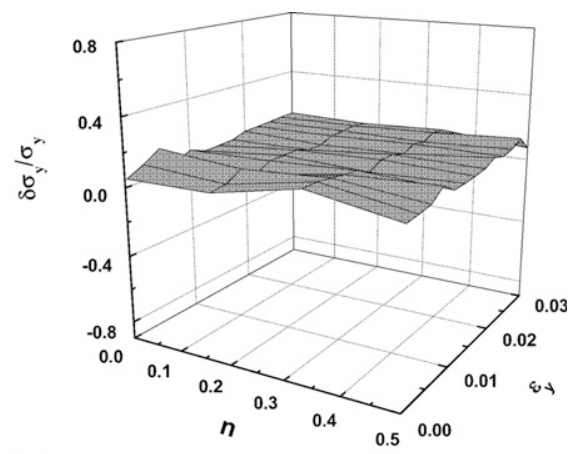

(b)

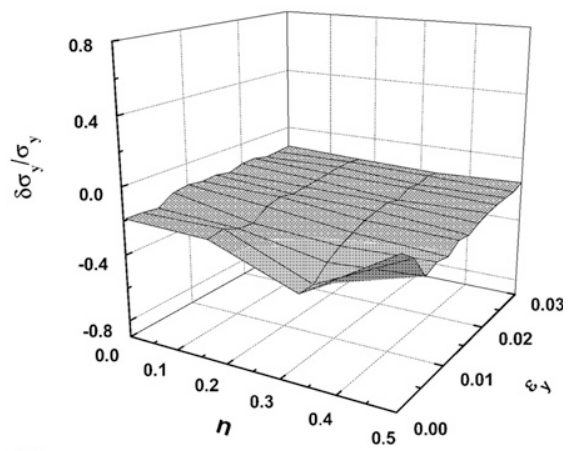

(d)

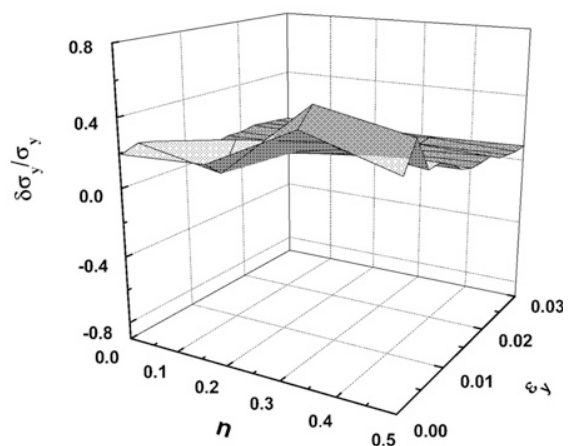

(f) 
into the sensitivity analysis varied linearly with the indentation depth $(a / R \geqslant 0.15$ and $h / R \leqslant 0.12)$ as follows: (i) from 0 to $3 \%$; (ii) from $-3 \%$ to 0 ; (iii) from $3 \%$ to 0 ; and (iv) from 0 to $-3 \%$. By introducing such perturbation, both the global error and the shape error of the load-depth curve can be evaluated. The resulting deviation of yield strength is presented in Fig. 6. It can be clearly seen that the maximum deviation of yield strength is $\sim 200 \%$, but in most cases, the deviation is $<30 \%$.

For method (V), we can derive more stable results. The maximum deviation of yield strength caused by the uncertainties in $m$ is $\sim 30 \%$, and in most cases, these deviations are $<10 \%$ (Fig. 7).

It should be noted that the sensitivity distributions of these methods are similar. The highest sensitivity is always observed for the material with plastic properties combination $\varepsilon_{y}=0.0003$ and $n=0.5$. With increasing $\varepsilon_{y}$ or decreasing $n$, the sensitivity of the methods tends to decrease. If the criteria is adopted that the maximum error and the deviation of yield stress should be $<20$ and $30 \%$, respectively, the validity ranges of these methods can be summarized as shown in Fig. 8 .

The horizontal abscissa of Figs. 8(a) and 8(b) are, respectively, logarithmic coordinate and normal coordinate, which enable us to clearly observe the difference of validity ranges of these methods for large and small $\varepsilon_{y}$, respectively. From Fig. 8, we can conclude that method (V) can give accurate and stable predicted results for all of the materials examined in this paper, methods (II) and (III) have smaller ranges of validity, and an even smaller range of validity is found for method (IV).

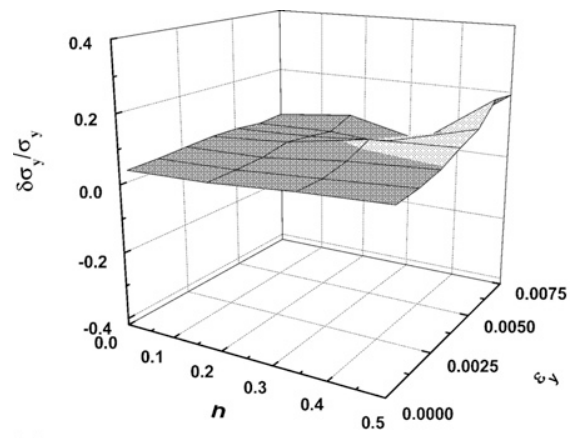

(a)

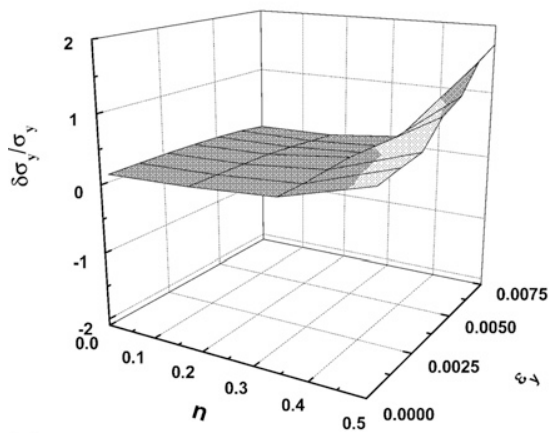

(c)

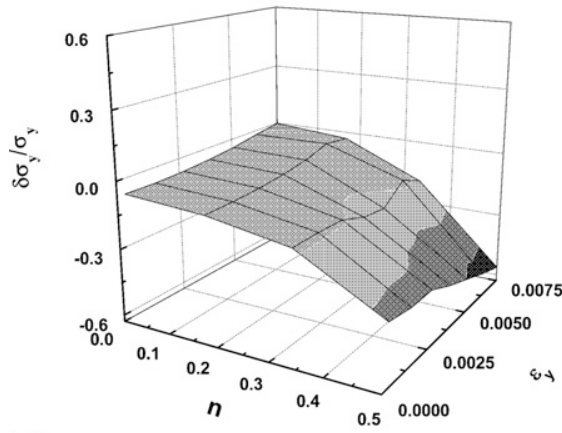

(e)

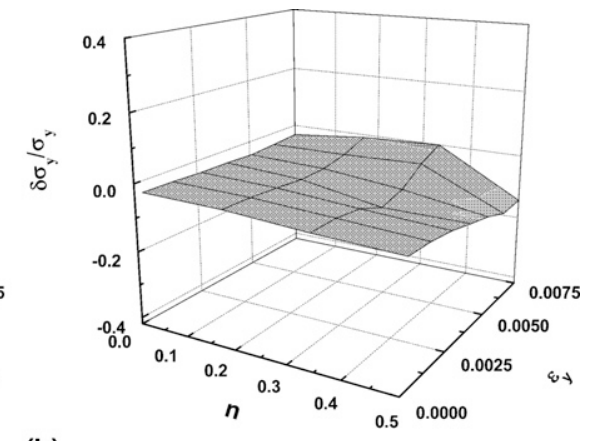

(b)

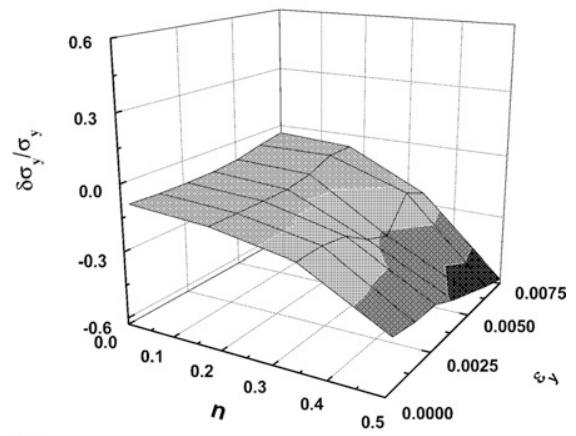

(d)

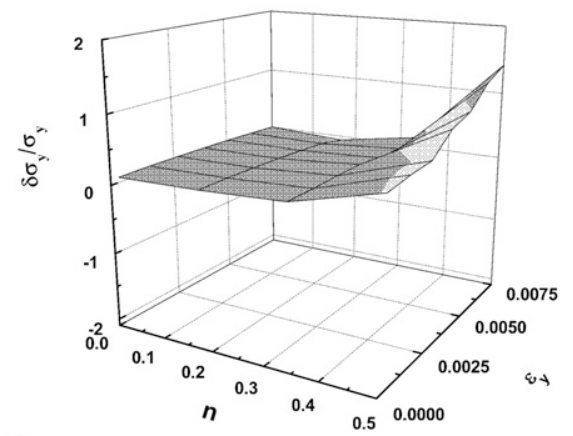

(f)

FIG. 6. Sensitivity distribution observed in determination of the yield stress of method (IV) with uncertainties of (a, b) $+4 \%$ and $-4 \%$ in $E$; (c, d) from $+3 \%$ to 0 and from $-3 \%$ to 0 in $F$; and (e, f) from 0 to $+3 \%$ and from 0 to $-3 \%$ in $F$. 


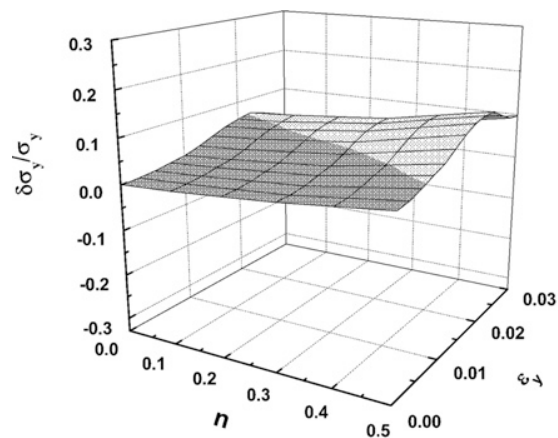

(a)

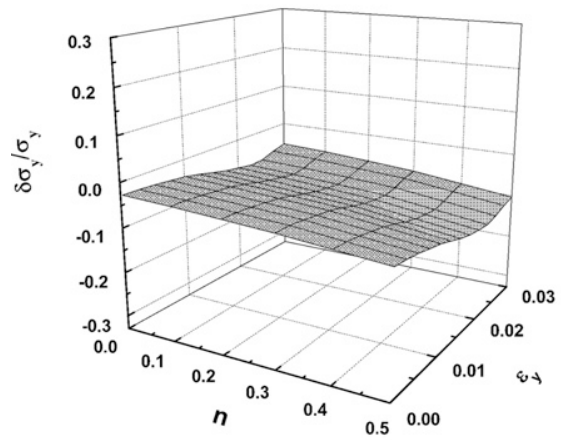

(c)

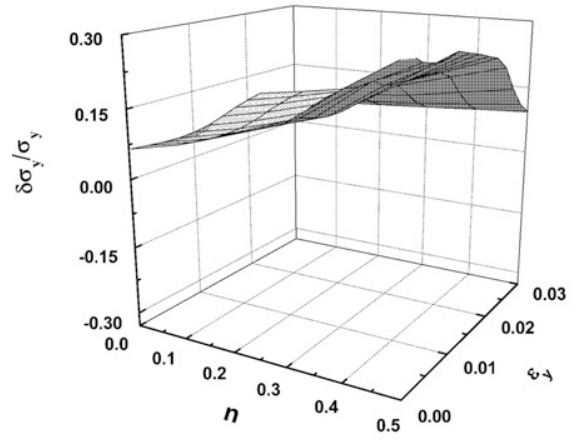

(e)

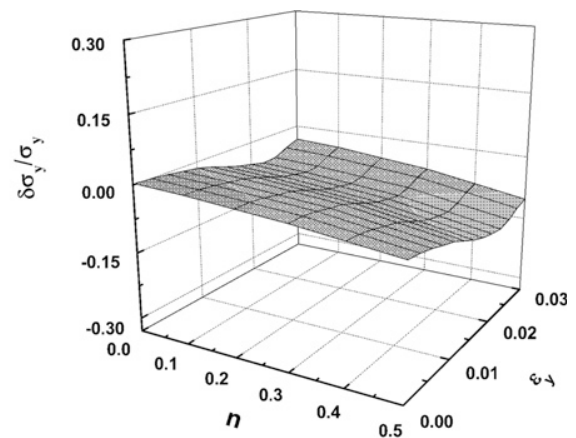

(b)

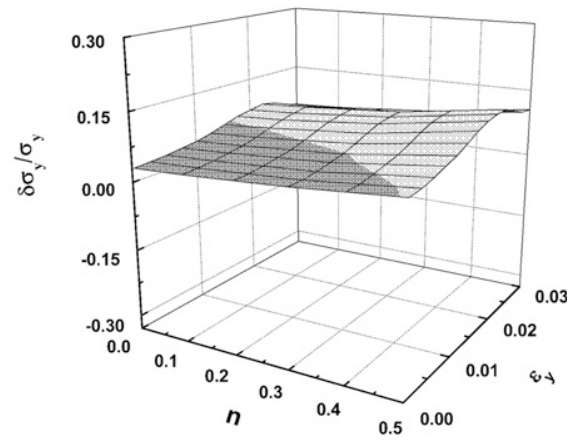

(d)

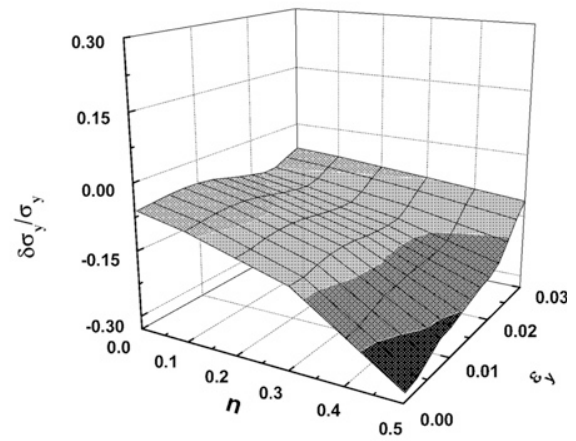

(f)

FIG. 7. Sensitivity distribution observed in determination of the yield stress of method (V) with uncertainties of $(\mathrm{a}, \mathrm{b})+4 \%$ and $-4 \%$ in $E$; (c, d) $+3 \%$ and $-3 \%$ in $W_{t}$; (e, f) and $+2 \%$ and $-2 \%$ in $m$.
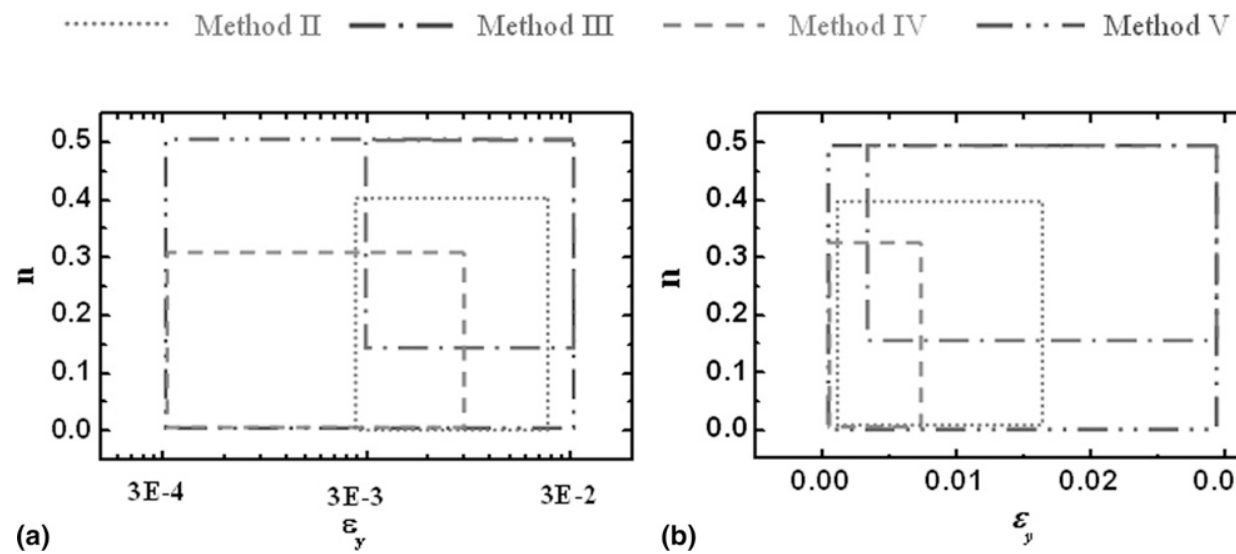

(a)

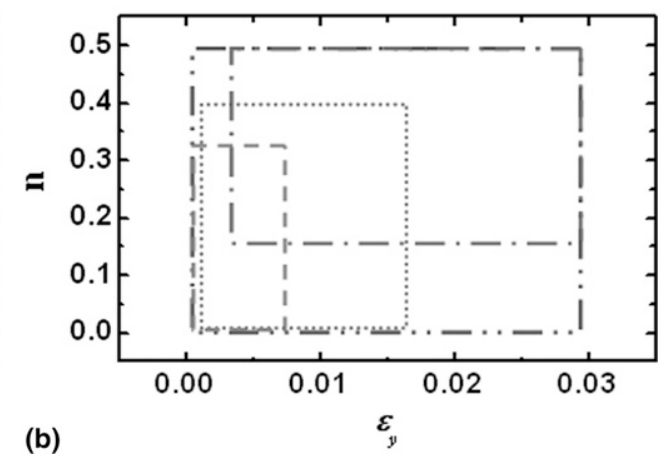

FIG. 8. The validity range of each method with the criteria that the error of predicted yield strength is $<20 \%$ and the deviation of yield strength caused by uncertainties of indentation responses is $\leq 30 \%$ : (a) logarithmic coordinate and (b) normal coordinate for the horizontal abscissa. 


\section{CONCLUSION}

From the numerical results discussed above, we can conclude that method (I) cannot be used to derive reasonable results, and no correlation can be found between its predicted results and the exact values of materials plastic properties. The suggestion of Herbert et al. ${ }^{25}$ of the constraint factor in this model being 3.7 also does not seem persuasive.

Methods (II) and (IV) are applicable only for materials within a certain range of properties, and the maximum relative errors of yield strength predicted by these two methods are $>50$ and $80 \%$, respectively. In contrast, methods (III) and (V) can give unique estimations for all verified materials, and the maximum predicted errors are $\sim 40$ and $10 \%$, respectively.

Methods (II) and (III) exhibit similar sensitivity character. The maximum deviation of yield strength is $\sim 60 \%$ by introducing the above-mentioned perturbations in the indentation responses. Even higher sensitivity was found for method (IV), whose maximum deviation of yield strength is $\sim 200 \%$. However, all of these methods, except for method (I), can give relative stable results in a certain range of properties. In contrast, method $(\mathrm{V})$ can deliver more stable and accurate results for all of the materials verified in this work.

By adopting the criteria that the predicted error is $<20 \%$ and the deviation of yield stress caused by uncertainties of indentation responses introduced in this paper is $\leqslant 30 \%$, the validity ranges of these models, except for method (I), are assessed as follows: method (II), $0.0020 \leqslant \varepsilon_{y} \leqslant 0.0120$ and $0 \leqslant n \leqslant 0.5$; method (III), $0.0030 \leqslant \varepsilon_{y} \leqslant 0.0300$ and $0.1 \leqslant n \leqslant 0.5$; method (IV), $0.0003 \leqslant \varepsilon_{y} \leqslant 0.0050$ and $0 \leqslant n \leqslant 0.5 ; \operatorname{method}(\mathrm{V})$, $0.0003 \leqslant \varepsilon_{y} \leqslant 0.0300$ and $0 \leqslant n \leqslant 0.5$.

It must be stressed that all of above results are derived based on the power law hardening model. However, the real material stress-strain curve is always different from the ideal model. As shown in the study of Liu et al., ${ }^{31}$ such a difference will also introduce predicted errors in these methods, but the extent to which this will affect the accuracy of these methods, for real materials, is still unresolved.

It should also be noted that all five methods adopt different relative indentation depths $(h / R)$ for analysis, which results in different applicable experimental conditions. For example, the smaller the relative depth, the larger the indenter radius that is needed to overcome the effects of surface roughness, grain size, the method of surface polishing that was used, and so on.

To clarify these issues, it is also necessary to perform experimental verification to examine their effectiveness and determine which method has the least requirements for experimental conditions. Further study will focus on this topic.

\section{ACKNOWLEDGMENT}

We acknowledge the support of the National Natural Science Foundation of China (Grant Nos. 10872200, 10721202, and 10572142).

\section{REFERENCES}

1. N. Choppacoop, M. Dao, and S. Suresh: Depth-sensing instrumented indentation with dual sharp indenters. Acta Mater. 51. 3713 (2003).

2. Y.T. Cheng and C.M. Cheng: Can stress-strain relationships be obtained from indentation curves using conical and pyramidal indenters? J. Mater. Res. 14, 3493 (1999).

3. H.Z. Lan and T.A. Venkatesh: On the sensitivity characteristics in the determination of the elastic properties of materials through multiple indentation. J. Mater. Res. 22, 1043 (2007).

4. Y.P. Cao and J. Lu: An energy-based method to extract plastic properties of metal materials from conical indentation tests. J. Mater. Res. 20. 1194 (2005).

5. N. Ogasawara, N. Chiba, and X. Chen: Representative strain of indentation analysis. J. Mater. Res. 20, 2225 (2005).

6. L. Wang, M. Ganor, and S.I. Rokhlin: Inverse scaling functions in nanoindentation with sharp indenters. Determination of material properties. J. Mater. Res. 20, 987 (2005).

7. D. Tabor: Hardness of Metals (Clarendon Press, Oxford, UK, 1951).

8. K.L. Murty, M.D. Mathew, Y. Wang, V.N. Shah, and F.M. Haggag: Nondestructive determination of tensile properties and fracture toughness of cold worked A36 steel. Int. J. Press. Vessels Pip. 75, 831 (1998).

9. J.H. Ahn and D. Kwon: Derivation of plastic stress-strain relationship from ball indentation: Examination of strain definition and pileup effect. J. Mater. Res. 16, 3170 (2001).

10. J.Y. Kim and K.W. Lee: Determination of tensile properties by instrumented indentation technique: Representative stress and strain approach. Surf. Coat. Technol. 201, 4278 (2006).

11. H. Lee: Ball indenter utilizing FEA solutions for property evaluation. U.S. Patent No. WO 03/010515 A1.

12. S. Jayaraman, G.T. Hahn, W.C. Oliver, C.A. Rubin, and P.C. Bastias: Determination of monotonic stress strain curve of hard materials from ultra-low-load indentation tests. Int. J. Solids Struct. 35, 365 (1998)

13. B. Taljat, T. Zacharia, and F. Kosel: New analytical procedure to determine stress-strain curve from spherical indentation data. Int. J. Solids Struct. 35, 4411 (1998)

14. J.S. Field and M.V. Swain: Determining the mechanical properties of small volumes of material from submicron spherical indentations. J. Mater. Res. 10, 101 (1995).

15. S. Kucharski and Z. Mroz: Identification of plastic hardening parameters of metals from spherical indentation tests. Mater. Sci. Eng. A 318,65 (2001).

16. E.G. Herbert, G.M. Pharr, and W.C. Oliver: On the measurement of tress-strain curves by spherical indentation. Thin Solid Films 398-399, 331 (2001).

17. W.P. Yu and J.P. Blanchard: An elastic-plastic indentation model and its solutions. J. Mater. Res. 11, 2358 (1996).

18. Y.P. Cao and J. Lu: A new method to extract the plastic properties of metal materials from an instrumented spherical indentation loading curve. Acta Mater. 52, 4023 (2004).

19. M.H. Zhao, N. Ogasawara, N. Chiba, and X. Chen: A new approach to measure the elastic-plastic properties of bulk materials using spherical indentation. Acta Mater. 54, 23 (2006). 
20. M. Beghini, L. Bertini, and V. Fontanari: Evaluation of the stressstrain curve of metallic materials by spherical indentation. $\underline{\text { Int }}$. J. Solids Struct. 43, 2441 (2006).

21. N. Huber and C. Tsakmakis: Determination of constitutive properties from spherical indentation data using neural networks. Part II: Plasticity with nonlinear isotropic and kinematic hardening. J. Mech. Phvs. Solids 47, 1589 (1999).

22. A. Nayebi, R. El Abdi, O. Bartier, and G. Mauvoisin: New procedure to determine steel mechanical parameters from the spherical indentation technique. Mech. Mater. 34, 243 (2002).

23. P. Jiang, T.H. Zhang, Y.H. Feng, and N.G. Liang: Determination of plastic properties by instrumented spherical indentation: Expanding cavity model and similarity solution approach. $\underline{. M a-}$ ter. Res. 24. 1045 (2009).

24. B. Guelorget, M. Francois, C. Liu, and J. Lu: Extracting the plastic properties of metal materials from microindentation tests: Experimental comparison of recently published methods. $\mathrm{J.Ma}$ ter. Res. 22, 1512 (2007).
25. E.G. Herbert, W.C. Pharr, and G.M. Oliver: On the measurements of yield strength by spherical indentation. Philos. Mag. 86, 5521 (2006).

26. Y.T. Cheng and C.M. Cheng: Scaling, dimensional analysis and indentation measurements. Mater. Sci. Eng. 44, 91 (2004).

27. R. Hill, B. Storakers, and A.B. Zdunek: A theoretical study of the Brinell hardness test. Proc. R. Soc. London. Ser. A 423, 301 (1989).

28. M. Dao and N. Chollacoop: Computational modeling of the forward and reverse problems in instrumented sharp indentation. Acta Mater. 49, 3899 (2001).

29. M. Mata and J. Alcala: The role of friction on sharp indentation. J. Mech. Phvs. Solids 52, 145 (2004).

30. W.C. Oliver and G.M. Pharr: An improved technique for determining hardness and elastic modulus using load and displacement sensing indentation experiments. J. Mater. Res. 7,1546 (1992).

31. L. Liu, N. Ogasawara, N. Chiba, and X. Chen: Can indentation technique measure unique elastoplastic properties? J. Mater. Res. 24, 784 (2009). 